\title{
An improved influence function method for predicting subsidence caused by longwall mining operations in inclined coal seams
}

\author{
Yi Luo ${ }^{1}$
}

Received: 31 March 2015/Revised: 30 June 2015/ Accepted: 31 July 2015/Published online: 22 September 2015

(C) The Author(s) 2015. This article is published with open access at Springerlink.com

\begin{abstract}
Prediction of surface subsidence caused by longwall mining operation in inclined coal seams is often very challenging. The existing empirical prediction methods are inflexible for varying geological and mining conditions. An improved influence function method has been developed to take the advantage of its fundamentally sound nature and flexibility. In developing this method, the original Knothe function has been transformed to produce a continuous and asymmetrical subsidence influence function. The empirical equations for final subsidence parameters derived from collected longwall subsidence data have been incorporated into the mathematical models to improve the prediction accuracy. A number of demonstration cases for longwall mining operations in coal seams with varying inclination angles, depths and panel widths have been used to verify the applicability of the new subsidence prediction model.
\end{abstract}

Keywords Subsidence prediction · Influence function method · Inclined coal seam · Longwall mining

\section{Introduction}

Longwall mining in inclined coal seams has been a common practice in many major coal mining countries. The characteristics of the subsidence basin induced by longwall operations in inclined coal seam are different from that caused by mining in a level seam. Accurately predicting final surface subsidence over such mining operations has been a challenge in the field of subsidence research. Most of the existing subsidence prediction methods for inclined seam are empirical type such as graphical and profile function methods. They are site specific and inflexible in dealing with the variations in geological and mining conditions. As a trend in developing subsidence prediction tools, the more versatile influence function methods (IFM) is considered to be more suitable in developing subsidence prediction method for mining operations conducted in

Yi Luo

yi.luo@mail.wvu.edu

1 Department of Mining Engineering, West Virginia University, Morgantown, WV, USA inclined coal seams. Kratzsch (1983) briefly listed some methods developed based on the IFM concept in predicting final subsidence. The more practical one, the zone area method, requires significant manual calculations and results in insufficient accuracy. The author has previously proposed one analytical method based on the IFM concept (Luo and Cheng 2009). A piece-wise asymmetric influence function depending on the seam inclination angle has been proposed. Based on the influence function, a mathematical model to evaluate the final surface movements and deformations has been developed. However, the piece-wise influence function can result in abnormal discontinuities in the resulting final movement and deformations at some points and the computation process is also cumbersome.

As an improvement to the previous mathematical model, a new influence function is proposed using an approach to mathematically project the original influence function for flat coal seam onto the inclined coal seam. The resulting influence function meets all essential requirements for its definition and produces continuous final surface movements and deformations.

In this paper, the development process of the new influence function has been detailed. For the completeness 
of the method, the essential mathematical derivations in the previous paper are modified and presented. The mathematical formulae for evaluating the final surface movements and deformations are given. The validity of the new model is demonstrated with some examples.

\section{Proposed influence function}

The two fundamental steps of employing influence function methods in subsidence prediction are: (1) definition of the influence function that describes the distribution of subsidence influence on the ground surface caused by the extraction of one element of the coal seam, and (2) integration of the influence function over the "mine area". A proper form of influence function should be carefully defined so that it can well represent the mechanism involved in the subsidence process.

In this paper, the original form of the influence function for subsidence caused by the extraction in flat coal seam of the Knothe's Theory [Eq. (1)] is modified to represent the asymmetrical subsidence influence along the seam dipping direction. In the equation, $x^{\prime}$ is the horizontal distance in a local coordinate system to be explained later. The $S_{\max }$ and $R$ are the maximum possible subsidence and radius of major influence, respectively. The definitions of these two important subsidence parameters have been given in other publications (Luo 1989; Peng and Luo 1992; Peng et al. 1995)

$f_{s}\left(x^{\prime}\right)=\frac{S_{\max }}{R} \mathrm{e}^{-\left(x^{\prime} / R\right)^{2}}$

Figure 1 shows the proposed scheme of applying influence function method in predicting final surface subsidence caused by longwall mining in an inclined coal seam. A

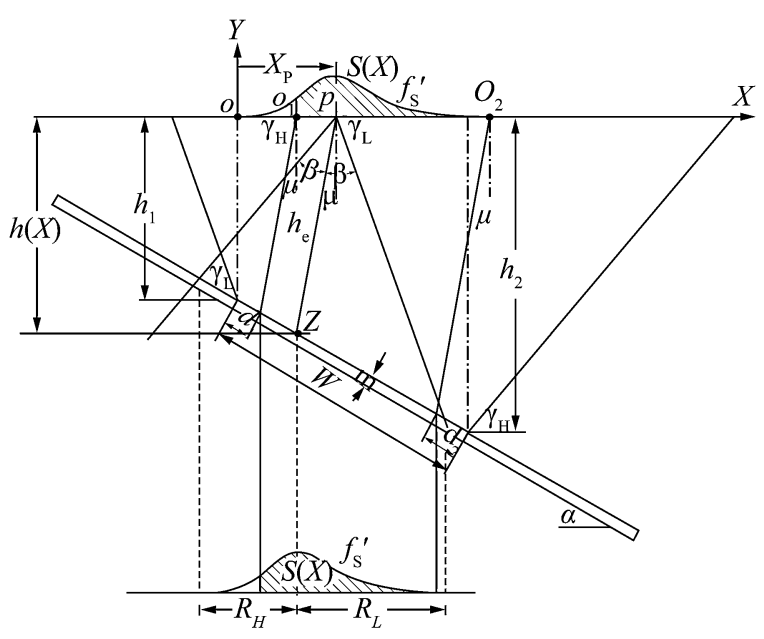

Fig. 1 Relations for using influence function method to predict final surface subsidence over a longwall gob in inclined coal seam convention is followed to establish the global coordinate system $(O-X)$ in which the origin $(O)$ is located directly above the left (upper) panel edge and its positive direction points to the right (lower) panel edge side. The surface point $(P)$ where the final surface subsidence is to be predicted is located $x_{\mathrm{p}}$ distance from the origin of the global coordinate system. A number of important parameters involved in defining the influence function are:

(1) The limit angles on the lower and upper sides of the panel $\left(\gamma_{\mathrm{H}}\right.$ and $\left.\gamma_{\mathrm{L}}\right)$, respectively. When they are plotted upwards from the lower and upper edges of the panel, they specify the edges of the final subsidence basin, respectively. When they are plotted from a surface point downward, they delineate the zone in which the extraction in the coal seam would influence the surface point to subside. These two lines are the lower and upper influence boundary lines. Generally, the limit angles depend on the angle of the seam inclination, $\alpha$. Rom (1964) developed a graph to determine $\gamma_{H}$ and $\gamma_{L}$ based on German subsidence experience. Chinese also derived their own empirical formulae for the limit angles for a number of mining districts (Fengfeng Subsidence Research Group 1982).

(2) Nadir angle $(\mu)$ shows the spatial relation between a specified surface point (point $P$ in Fig. 1) and the extraction point in the coal seam that influences surface point $\mathrm{P}$ to subside the most amount (point $Z$ ). It should be noted that the $P-Z$ line equally divides the angle formed between the lower and upper influence boundary lines drawn downwards from the surface point $\mathrm{P}$. The nadir angle is determined by

$\mu=\frac{1}{2}\left(\gamma_{\mathrm{L}}-\gamma_{\mathrm{H}}\right), \mathrm{deg}$

The effective radii of influence function $\left(R_{\mathrm{L}}\right.$ and $\left.R_{\mathrm{H}}\right)$ on lower and upper sides of the point of maximum extraction influence $(Z)$ are shown in Fig. 1. They can be determined by finding the intersection points between the inclined coal seam and the lower and upper influence boundary lines, respectively. The line equations for the lower and upper major influence boundaries $\left(\gamma_{\mathrm{L}}\right.$ and $\left.\gamma_{\mathrm{H}}\right)$ that intersect the prediction point at $x_{\mathrm{p}}$ are shown in Eqs. (3) and (4), respectively.

$y=\left(x_{\mathrm{p}}-x\right) \tan \gamma_{\mathrm{L}} \quad x>x_{\mathrm{p}}$

$y=\left(x-x_{\mathrm{p}}\right) \tan \gamma_{\mathrm{H}} \quad x \leq x_{\mathrm{p}}$

The line equation for the inclined coal seam is defined by Eq. (5) where $h_{1}$ is the overburden depth at upper panel edge.

$y=-h_{1}-x \tan \alpha$ 
The coordinates of the intersection point between the lower influence boundary and the coal seam are determined from Eqs. (3) and (5) as:

$x_{\mathrm{L}}=\frac{x_{\mathrm{p}} \tan \gamma_{\mathrm{L}}+h_{1}}{\tan \gamma_{\mathrm{L}}-\tan \alpha}$

$y_{\mathrm{L}}=\left(x_{\mathrm{p}}-x_{\mathrm{L}}\right) \tan \gamma_{\mathrm{L}}$

Similarly, the coordinates of the intersection point between upper influence boundary and the coal seam are determined as:

$$
\begin{aligned}
& x_{\mathrm{H}}=\frac{x_{\mathrm{p}} \tan \gamma_{\mathrm{H}}-h_{1}}{\tan \alpha+\tan \gamma_{\mathrm{H}}} \\
& y_{\mathrm{H}}=\left(x_{\mathrm{H}}-x_{\mathrm{p}}\right) \tan \gamma_{\mathrm{H}}
\end{aligned}
$$

The location of the maximum extraction influence point $(Z)$ is determined by finding the intersection point between the line equation of $P-Z$ and that of the inclined coal seam. The coordinates of the maximum extraction influence point are:

$x_{\mathrm{Z}}=\frac{\frac{x_{\mathrm{p}}}{\tan \mu}-h_{1}}{\frac{1}{\tan \mu}+\tan \alpha}$

$y_{Z}=-h_{1}-x_{Z} \tan \alpha$

The effective radii of major influence on the lower and upper sides can be simply determined using Eqs. (12) and (13), respectively. These radii of major influence are dependent on $x_{\mathrm{p}}, \alpha, \gamma_{\mathrm{L}}, \gamma_{\mathrm{H}}$ and overburden depth.

$$
\begin{aligned}
& R_{L}=x_{L}-x_{Z} \\
& R_{H}=x_{Z}-x_{H}
\end{aligned}
$$

The other parameter to define the influence function is the maximum possible subsidence, $S_{\max }$. In general, $S_{\max }$ decreases as the overburden depth increases. Therefore, $S_{\max }$ varies with $x_{\mathrm{p}}$. It is reasonable to assume that for an inclined coal seam $S_{\max }$ at a prediction point is a function of the effective distance $\left(h_{\mathrm{e}}\right)$ instead of the true depth. The effective distance is simply calculated from the coordinates of the prediction point $\left(x_{\mathrm{p}}, 0\right)$ and the point of maximum extraction influence $\left(x_{Z}, y_{Z}\right)$.

$h_{\mathrm{e}}=\sqrt{\left(x_{Z}-x_{\mathrm{p}}\right)^{2}+y_{Z}^{2}}$

The true subsidence factor can be estimated by substituting $h_{\mathrm{e}}$ into the empirical equation derived previously by the author from the collected US and Australian longwall subsidence data (Luo and Peng 2000). It should be noted that the unit of the effective distance $h_{e}$ in the following empirical equation is $\mathrm{ft}$ and one foot is $0.3048 \mathrm{~m}$.

$a=1.9381\left(h_{\mathrm{e}}+23.4185\right)^{-0.1884}$
The maximum possible subsidence at surface point $x_{\mathrm{p}}$ for an inclined coal seam is determined by Eq. (16).

$S_{\max }=a m \cos \alpha$

The influence function for subsidence in Knothe's theory (Knothe 1957) proven to be accurate for mining in flat seam [Eq. (1)] should be modified to represent the distribution of subsidence influence due to the extraction in an inclined coal seam according to a special transformation scheme. The transformation involves a mathematical projection of the symmetric influence function for flat seam, $f_{s}$, onto the inclined seam to form the new asymmetric influence function of $f_{s}^{\prime}$ as shown in Fig. 2. It should be noted that local coordinate systems are used in the transformation of the influence functions. For the flat seam, the local coordinate system is $o^{\prime}-x^{\prime}$ while that for the inclined seam is $o^{\prime}-\xi^{\prime}$.

The projection starts from point $(P)$ on ground surface. The first step is to project the influence boundaries for the upper and lower sides to the flat and inclined seams, respectively. When they intersect with the flat seam, the resulting radii of influence on both sides of the origin of the local coordinate system $o$ ' are the same as $R$. However, the resulting radii of major influence of the intersections with the inclined seam are different. On the upper side, the radius is $R_{\mathrm{H}}$ which is smaller than $R$ while that on the lower side $R_{\mathrm{L}}$ is larger than $R$.

To confirm with influence function definition, the first two essential requirements for the mathematical projection should be met: (1) the total area under the influence function $f_{s}$ is equal to that under $f_{s}^{\prime}$; (2) the area under each

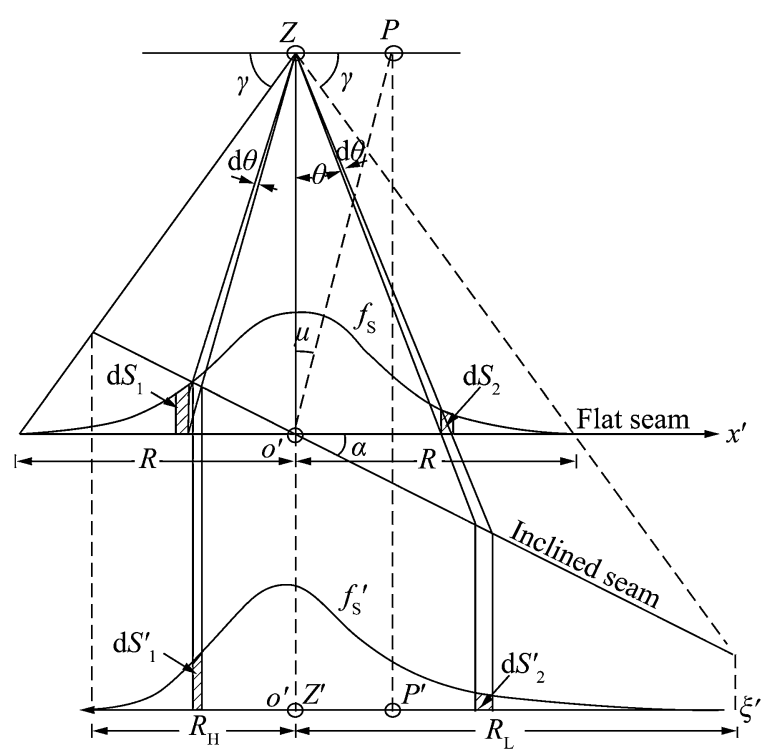

Fig. 2 Schematic of transforming influence function from flat seam to inclined seam 
half of the influence functions on the left side of the origin $\mathrm{o}^{\prime}$ should be equal to that on the right side.

In the second step of defining the influence function, an incremental angle $\mathrm{d} \theta$ is drawn from the projection angle $\theta$ as shown in Fig. 2. The left and right boundary lines of $\mathrm{d} \theta$ intersect the flat and inclined seams at different depths. The resulting widths along the horizontal direction for the intersections are different on the flat and inclined seams. Apparently, on the upper side the origin $o^{\prime}$, the width of the intersection on inclined seam is smaller than that on the flat seam while on the lower side where a reversed phenomenon is observed. The change in width can be more precisely described by the transformation of the horizontal coordinate in the respective local coordinate systems from $x^{\prime}$ to $\xi^{\prime}$ in Eq. (17). In the equation, $h$ is the actual overburden depth and $\theta=\tan ^{-1}\left(x^{\prime} / h\right)$. Apparently, the transformed local coordinate $\xi^{\prime}$ in the inclined seam depends on the seam inclination angle $\alpha$, overburden depth $h$ and the local coordinate $x^{\prime}$.

$\xi=\frac{x^{\prime} h}{\sqrt{x^{\prime 2}+h^{2}}} \frac{\cos \alpha}{\sin \left[180^{\circ}-\alpha-\tan ^{-1}\left(\frac{x^{\prime}}{h}\right)\right]}$

The transformation of the influence function must ensure that the elemental influence for the flat seam, $\mathrm{d} S_{2}$ as shown in Fig. 2, is equal to that for the inclined seam, $\mathrm{d} S_{2}^{\prime}$ - the third essential requirement. To meet this requirement, adjustments must be made on the height or value of $f_{s}$ ccording to the change in intersection widths. This adjustment is accomplished through the dividing the original influence function $f_{s}\left(x^{\prime}\right)$ in Eq. (1) by the first derivative of $\xi^{\prime}$ [Eq. (18)] with respect to $x^{\prime}$. The derivative term is shown in Eq. (19).

$f_{s}^{\prime}\left(\xi^{\prime}\right)=\frac{f_{s}\left(x^{\prime}\right)}{\frac{\mathrm{d} \xi^{\prime}}{\mathrm{d} x^{\prime}}}$

$\frac{\mathrm{d} \xi^{\prime}}{\mathrm{d} x^{\prime}}=\frac{h^{2}[1+\cos (2 \alpha)]}{2\left[\alpha+\tan ^{-1}\left(\frac{x^{\prime}}{h}\right)\right]\left(h^{2}+x^{\prime 2}\right)}$

The new subsidence influence function is then defined using Eqs. (18) and (19). Both Eqs. (1) and (19) are continuous and Eq. (19) has no zero point in the range from $-\infty$ to $\infty$. The features ensure the new subsidence influence function to be continuous through the same range-an important requirement to result in continuous final surface movements and deformations.

Based on the proposed transformation, the new influence functions for a number of seam inclination angles (i.e., $\alpha=0^{\circ}, 15^{\circ}, 25^{\circ}, 35^{\circ}$ and $45^{\circ}$ ) for an overburden depth of $h=800 \mathrm{ft}(244 \mathrm{~m})$ are plotted in Fig. 3. The continuous nature for each of the new influence function is apparent. The asymmetry of each influence function, skewing toward the upper panel edge side, becomes more severe as the angle of seam inclination increases. The peak of the

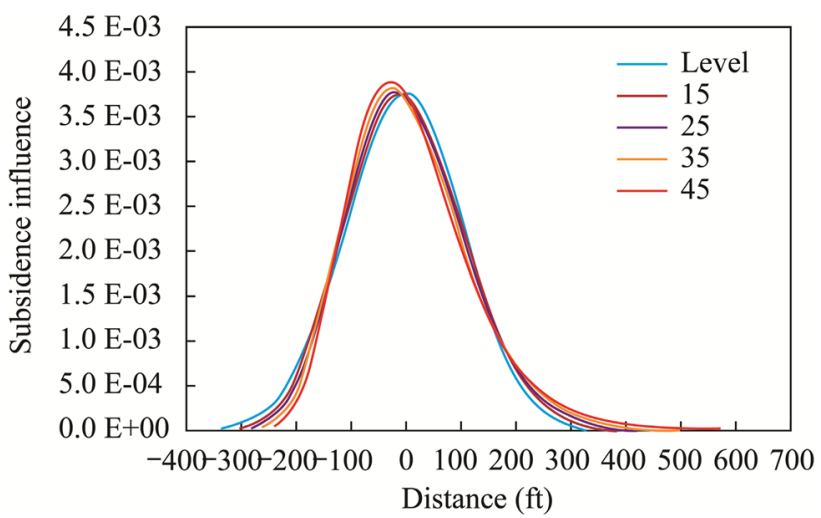

Fig. 3 Influence functions for various seam inclinations $(h=800 \mathrm{ft}$ or $244 \mathrm{~m})$

influence function moves toward the upper panel edge side and the magnitude increases slightly also with the inclination angle. Therefore, the transformed influence function meets all the essential requirements as stated previously.

\section{Final surface movements and deformations}

The final surface movements are obtained by integrating the influence functions. The final deformations are the derivatives of the final surface movements.

\subsection{Determination of final subsidence}

Based on the concept of the influence function method, the final subsidence at a surface point is the summation of all influences received at this point caused by the extraction in the coal seam within the influence boundaries. Mathematically, it is the integral of the subsidence influence function over the "mined" area. After considering the overhanging overburden strata over the panel edges and an equivalent transformation of the coordinate system, the final surface subsidence at the prediction point can be determined by integrating the transformed influence function between the left and right inflection points, $O_{1}$ and $O_{2}$ (Fig. 1). The offsets of the inflection points at the coal seam level $\left(d_{1}\right.$, and $d_{2}$ ) are first determined using the empirical formula [Eq. (20)] derived from the collected US subsidence data (Peng et al. 1995). The actual overburden depths on the left and right edges of the panel $\left(h_{1}\right.$ and $\left.h_{2}\right)$ are used in Eq. (20) to obtain the offsets of inflection points on the left and right sides of the panel, respectively. It should be noted that the units for the depth and offset distance in the empirical equation are $\mathrm{ft}$.

$d_{1,2}=h_{1,2}\left(0.382075 \times 0.999253^{h_{1,2}}\right)$ 
It is reasonable to project the two inflection points from the coal seam to the surface with the nadir angle $(\mu)$ since this angle signifies the line of the maximum influence on the ground surface caused by the underground extraction (Fig. 1). Through such projection, the coordinates of the left and right inflection points in the global coordinate system, $x_{1}$ and $x_{2}$, are determined as:

$\left\{\begin{array}{l}x_{1}=d_{1} \cos \alpha+\left(h_{1}+d_{1} \sin \alpha\right) \tan \mu \\ x_{2}=\left(W-d_{2}\right) \cos \alpha+\left(h_{2}-d_{2} \sin \alpha\right) \tan \mu\end{array}\right.$

In performing the integration of the influence function between the inflection points, the inflection points should be expressed in local coordinates.

$\left\{\begin{array}{l}\xi_{1}^{\prime}=x_{1}-x_{\mathrm{p}} \\ \xi_{2}^{\prime}=x_{2}-x_{\mathrm{p}}\end{array}\right.$

The Final subsidence at prediction point, $x_{\mathrm{p}}$, is shown in Fig. 1 as the shaded area and is determined as

$S\left(x_{\mathrm{p}}\right)=\int_{\xi_{1}^{\prime}}^{\xi_{2}^{\prime}} f_{s}^{\prime}\left(\xi^{\prime}\right) \mathrm{d} \xi^{\prime}$

\subsection{Determinations of final movement and deformations}

The other final surface movement (i.e., horizontal displacement) and deformations (i.e., slope, strain and curvature) along a major cross-section in the dipping direction of the inclined seam are directly related to or as the derivatives of the final surface subsidence. Since the depth at a given prediction point $h$ is dependent on the location $x$, obtaining the analytical expressions for the derivatives will be very cumbersome if it is possible. Numerical differentiation techniques are used to evaluate the final horizontal displacement, slope, strain and curvature. The final surface slope is defined as:

$i\left(x_{\mathrm{p}}\right)=\frac{\mathrm{d} S\left(x_{\mathrm{p}}\right)}{\mathrm{d} x_{\mathrm{p}}}$

Based on the traditional subsidence theories, the final horizontal displacement is proportional to the final slope. For a flat coal seam, the proportionality coefficient is defined as $R^{2} / h$ where $R$ is the radius of major influence and $h$ is the overburden depth (Luo 1989). It has been found that $R$ is normally one third of $h$ worldwide and the proportionality coefficient is $h / 9$. For mining in an inclined coal seam, the effective distance $h_{\mathrm{e}}$ replaces $h$ for determining the proportionality coefficient. Therefore, the horizontal displacement at the prediction point is defined as:

$U\left(x_{\mathrm{p}}\right)=\frac{h_{\mathrm{e}}\left(x_{\mathrm{p}}\right)}{9} i\left(x_{\mathrm{p}}\right)$
The final surface strain and curvature at the prediction point are the first derivatives of the final horizontal displacement and slope as shown in Eqs. (26) and (27), respectively.

$\varepsilon\left(x_{p}\right)=\frac{\mathrm{d}}{\mathrm{d} x_{\mathrm{p}}} U\left(x_{\mathrm{p}}\right)$

$K\left(x_{\mathrm{p}}\right)=\frac{\mathrm{d}}{\mathrm{d} x_{\mathrm{p}}} i\left(x_{\mathrm{p}}\right)$

\subsection{Verification of the new model}

In order to verify the proposed mathematical model, a computer program developed in MathCad is used to carry out the required computations of final surface subsidence prediction for longwall mining operations conducted in inclined coal seams. Figure 4 shows the predicted final subsidence profiles of a $1000 \mathrm{ft}(305 \mathrm{~m})$ wide longwall panel in a $5.5 \mathrm{ft}(1.68 \mathrm{~m})$ thick coal seam with different inclination angles (i.e., $0^{\circ}, 15^{\circ}, 30^{\circ}$ and $45^{\circ}$ ). The overburden depth at the upper panel edge is $600 \mathrm{ft}(183 \mathrm{~m})$. For mining conducted in level coal seam $\left(0^{\circ}\right.$ inclination angle), the final subsidence shows a super-critical final subsidence basin symmetric about the panel center. As the seam inclination increases, the asymmetric nature becomes severer with the shape of the subsidence basin on the lower half being gentler than that on the upper side. The maximum possible subsidence, $S_{\max }$, decreases as the inclination angle increases as a result of Eq. (16). The subsidence profiles also shift toward the lower side and more and more significant subsidence occurs beyond the lower panel edge. Different from the other three subsidence profiles, the one

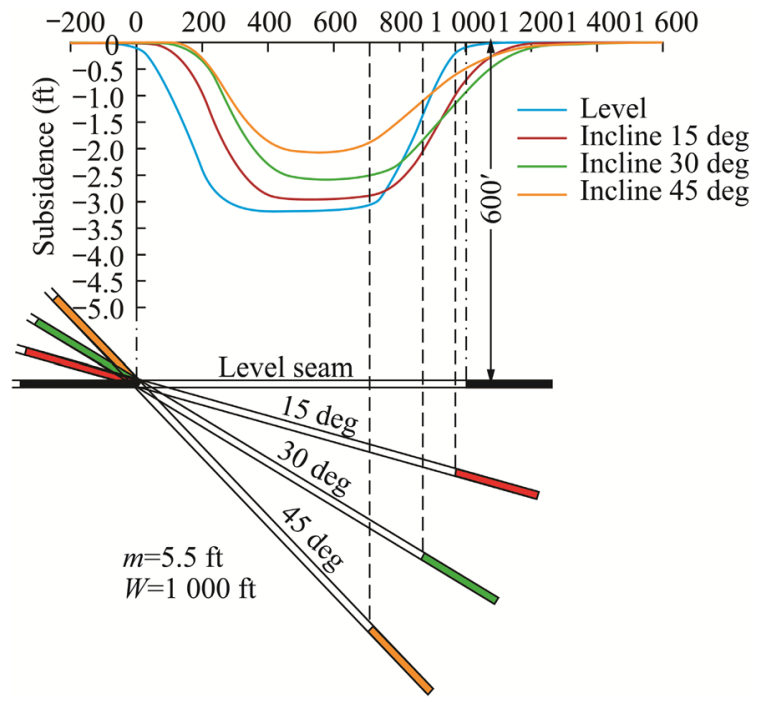

Fig. 4 Predicted final subsidence profiles over $1000 \mathrm{ft}(305 \mathrm{~m})$ wide panel in $5.5 \mathrm{ft}$ coal seam with different inclination angles 
for the $45^{\circ}$ inclined seam shows a subcritical subsidence basin.

Figure 5 shows three predicted final subsidence profiles over a $30^{\circ}$ inclined coal seam when the widths of the longwall panel are 800, 1000 and $1500 \mathrm{ft}(244,305$ and $457 \mathrm{~m})$, respectively. The mining height and the overburden depth at the upper panel edge are 7.0 and $700 \mathrm{ft}(2.13$ and $213 \mathrm{~m}$ ), respectively. The locations of the lower panel edges are also plotted in the figure. In addition to producing asymmetric subsidence profiles, the ability for the influence function method to adapt the change in panel width automatically from sub-critical ( $w=800 \mathrm{ft}$ or $244 \mathrm{~m}$ ), critical and super-critical ( $w=1500 \mathrm{ft}$ or $457 \mathrm{~m}$ ) conditions is evident. This is the most important advantage of the influence function methods over the empirical methods. In the supper-critical condition, a large "flat" subsidence basin bottom appears in the central portion of the basin. However, the maximum possible subsidence in the "flat" bottom is no longer an uniform amount as expected for flat coal seam but decreases slightly toward the lower side of the panel.

The most important improvement for introducing this new continuous influence function is to ensure the continuity of the resulting final horizontal displacement, slope, strain and curvature. Figure 6 shows the predicted final strain profiles for the same examples shown in Fig. 5. The three profiles show smooth and continuous nature throughout their respective entire ranges. The peak values of the maximum tensile and compressive strains decrease as the overburden increases.

The application examples in this section show the desirable attributes of surface final subsidence caused by longwall mining operations in inclined coal seam. The magnitudes and distributions of predicted final surface movements and deformations are reasonable. Therefore, the improved model based on the concept of influence function method is a good tool for subsidence prediction

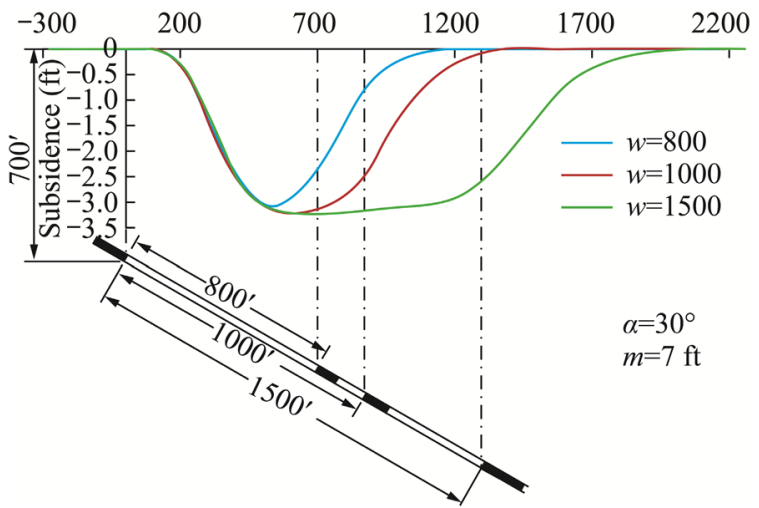

Fig. 5 Predicted final subsidence profiles for different panel widths in $30^{\circ}$ coal seam

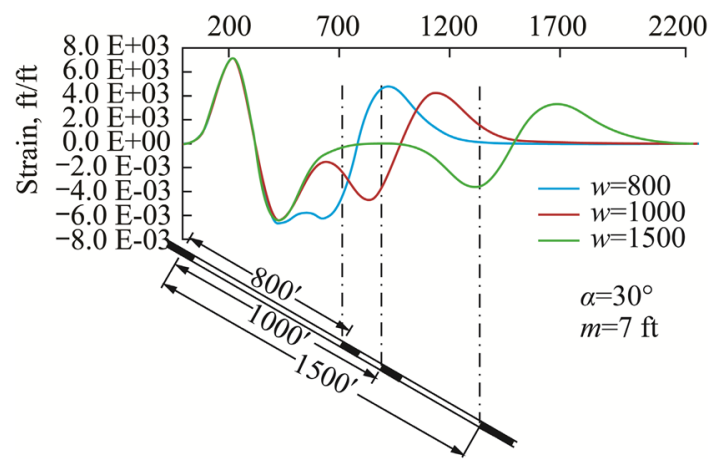

Fig. 6 Predicted final strain profiles for different panel widths in $30^{\circ}$ coal seam

caused by longwall mining operations in inclined coal seam.

\section{Case demonstrations}

In order to further demonstrate the capabilities of the improved mathematical model, two Chinese cases with moderate and steep coal seam inclinations are predicted using the developed model and presented in this section.

Figure 7 shows the predicted final surface and deformation profiles for a typical subsidence case in Jixi mining district. In this case, the coal seam dips toward the right at an angle of $18^{\circ}$. The depth on the upper panel edge is $215 \mathrm{ft}(65 \mathrm{~m})$. The mining height is $4.5 \mathrm{ft}(1.37 \mathrm{~m})$. The panel is $600 \mathrm{ft}(183 \mathrm{~m})$. Due to the relatively small depth, the resulting subsidence basin is a supercritical one. However, the flat bottom portion of a supercritical subsidence basin is not necessarily flat but the maximum subsidence there decreases as the depth increases. The subsidence profile on the deeper side is much gentler than that on the shallow side. Another observation is that the maximum horizontal displacement, tensile and

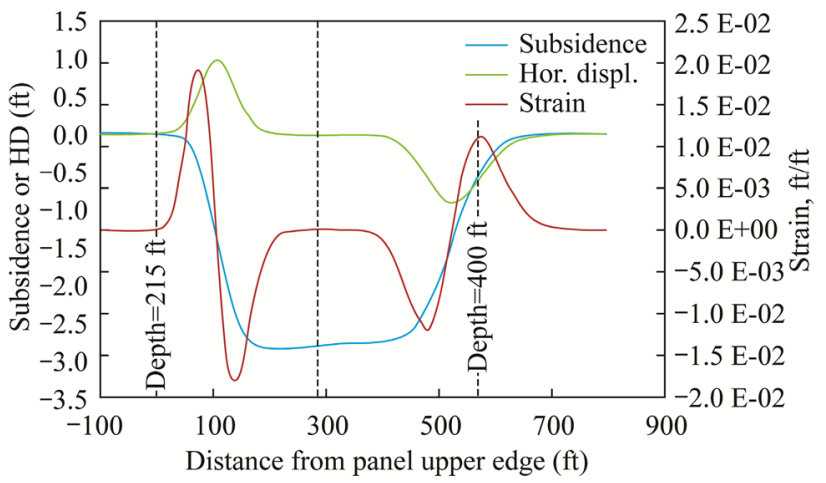

Fig. 7 Predicted final subsidence, horizontal displacement and strain profiles for Jixi mining district 


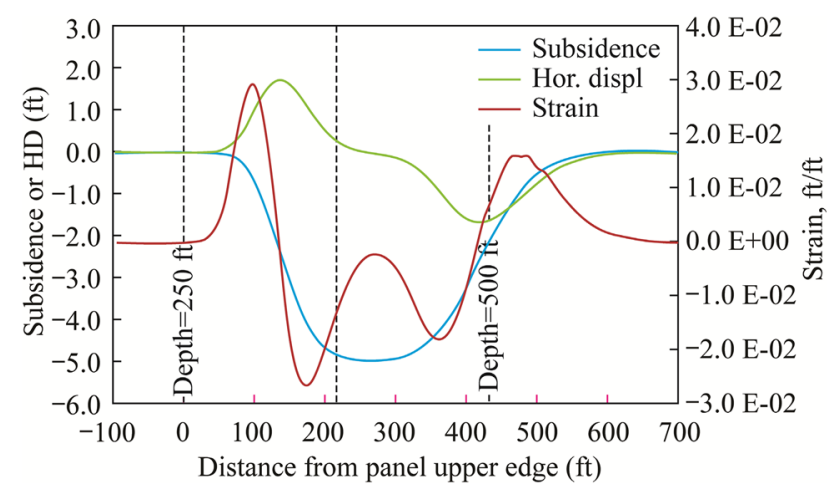

Fig. 8 Predicted final subsidence, horizontal displacement and strain profiles for Huainan mining district

compressive strains on the deeper side are smaller than those on the shallower side as expected.

Figure 8 shows the predicted final surface movement and deformation profiles typical for Huainan mining district. The geological and mining information includes: $\alpha=30^{\circ}, h_{1}=250 \mathrm{ft}(76.2 \mathrm{~m}), W=500 \mathrm{ft}(152 \mathrm{~m})$ and $m=9.0 \mathrm{ft}(2.74 \mathrm{~m})$. Due to the much larger mining height, the maximum movements and deformations are larger than those in the previous cases.

Compared to the previous model (Luo and Cheng 2009), the discontinuity problems at some prediction points are totally avoided in the predicted strain profile (similarly in slope, horizontal displacement and curvature profiles) using the new prediction method.

\section{Conclusions}

The mathematical model to employ influence function method for predicting final surface subsidence over a longwall mining operation in an inclined coal seam has been improved. The main improvement is to define a continuous asymmetric subsidence influence function to represent the influence to surface subsidence induce by extracting an element in an inclined coal seam. The degree of asymmetry of the influence function is dependent on the angle of the seam inclination. The determination of the final subsidence at a prediction point is performed through integrating the influence function between the inflection points. Once the final surface subsidence is determined, the other movement and deformations are obtained through numerical differentiations.

Based on the case demonstrations, the improved influence function method represents the characteristics of the final subsidence basin formed over longwall gob in an inclined coal seam well. Its flexibility to deal with varying seam inclination and degree of critical extraction makes the influence function method a much better tool in subsidence prediction than the existing methods. It provides a more accurate and faster tool to perform the complicated calculations for the final surface movements and deformations.

Open Access This article is distributed under the terms of the Creative Commons Attribution 4.0 International License (http://crea tivecommons.org/licenses/by/4.0/), which permits unrestricted use, distribution, and reproduction in any medium, provided you give appropriate credit to the original author(s) and the source, provide a link to the Creative Commons license, and indicate if changes were made.

\section{References}

Fengfeng Subsidence Research Group (1982) Research findings on subsidence characteristics in Fengfeng Mining District. Fengfeng Coal Sci Technol 3 (in Chinese)

Knothe S (1957) Observation of surface movements under influence of mining and their theoretical interpretation. In: Proceedings of the European congress of ground movements, Leeds, pp 210-218

Kratzsch H (1983) Mining subsidence engineering. Springer-Verlag, New York, p 543

Luo Y (1989) Integrated computer model for predicting surface subsidence due to underground coal mining-CISPM. PhD Dissertation, Morgantown: West Virginia University, 168. (UMI order No. 9020385)

Luo Y, Cheng JW (2009) An influence function method based subsidence prediction program for longwall mining operations in inclined coal seams. J Min Sci Technol 19(9):592-598

Luo Y, Peng SS (2000) Prediction of subsurface subsidence for longwall mining operations. In: Proceedings of the 19th international conference on ground control in mining, pp 163-170

Peng SS, Luo Y (1992) Comprehensive and integrated subsidence prediction model-CISPM (V2.0). In: Proceedings of the 3rd workshop on surface subsidence due to underground mining, Mor-gantown, pp 22-31

Peng SS, Luo Y, Zhang ZM (1995) Subsidence parameters - their definitions and determination. AIME-SME Trans 300:60-65

Rom H (1964) A limit angle system. Mitt Markscheidew 71:197-199 